\title{
O médico para Saúde Coletiva no Estado do Amazonas: lacunas na formação, lacunas na atenção
}

\author{
Physicians in public health in the Amazon: gaps in \\ undergraduate training and health services
}

Rodrigo Otávio Moretti-Pires ${ }^{\mathrm{I}}$

\author{
PALAVRAS-CHAVE \\ - Saúde pública \\ - Atenção primária à Saúde \\ - Ensino superior \\ - Medicina
}




\section{INTRODUÇÃO}

Nas duas últimas décadas, o setor público de saúde no Brasil tem-se colocado, sem dúvida, num processo caracterizado pela valorização de propostas inovadoras nos campos da assistência e da gestão pública - impulsionada pelos princípios de democracia e de justiça social que orientaram o movimento de reforma sanitária brasileira. Paradoxalmente, também vem sendo o lócus de manifestação das contradições entre teoria/prática e do intenso processo de exclusão, segregação e desvalorização da vida que tem marcado a sociedade brasileira.

O discurso governamental prima por firmar e confirmar que o espaço em que se dá a atenção pública à saúde no Brasil está ressignificado como momento de empoderamento e de fazer valer os direitos sociais do cidadão, em particular no que se refere a universalidade, integralidade e equidade como premissas ideológicas de como o Sistema Único de Saúde (SUS) deve se apresentar ao usuário que o acessa.

Neste enfoque, Campos $^{1}$ defende que o evento da procura do usuário pelo SUS não pode ser encarado tão-somente como a busca de solução para um problema de saúde, mas, sim, como um evento complexo, que envolve recorrer ao profissional médico e à unidade de saúde - seja na atenção primária, secundária ou terciária. O que decorrerá será o resultado em que interagem complexas e imbricadas relações, dependentes tanto da disponibilidade do conjunto de ações e serviços previstos pelo sistema, como das dificuldades de acesso aos mesmos - termo este que não se restringe somente ao acessar ou não os serviços, mas também ao acesso com qualidade e eficiência, ou não, havendo diversas nuances e graus de variação neste contexto, diretamente relacionado à implementação do princípio doutrinário da integralidade no SUS

Em especial, as mudanças implementadas no âmbito do SUS no que se refere a enfoque, processo de trabalho e compreensão do impacto das ações de saúde distanciam cada vez mais a atuação médica idealizada - principalmente quanto à Estratégia de Saúde da Família - e a formação médica corrente ${ }^{2,3}$. Isto porque, ainda nos dias atuais, a orientação deste profissional se pauta na especialização de cunho flexneriano e biomédico ${ }^{4}$, o que, fundamentalmente, foge do talhe oficial das reformulações em termos de políticas de saúde adotadas pelo Estado, que preconizam a assistência em um sistema público de saúde acessível aos usuários não apenas em termos de acesso físico ao serviço para as demandas biológicas e biologicistas, mas também em termos de acolhimento, humanização, solidariedade e resolutividade dos problemas de saúde, que, como consagrado amplamente na literatura, têm origem multifatorial e são direta e indiretamente influenciados pelo entorno sociocultural dos usuários do sistema.
Há incoerência entre este enfoque e o atendimento especializado, característico da formação médica tradicional no Brasil, na qual o hospital constitui lócus privilegiado para a aprendizagem profissional, comprometendo a construção de um profissional que paute sua prática nas premissas da atenção primária à saúde preconizadas pelo Estado ${ }^{5-9}$

Em época de reestruturação produtiva do capital, em que o mundo do trabalho enfrenta crise e mudança nas relações trabalhistas e nas oportunidades de emprego, contexto que também afeta as profissões tradicionalmente liberais do setor de prestação de serviços de saúde, a Saúde Coletiva e suas diversas frentes profissionais se tornam opções mais seguras e estáveis para a inserção do profissional no mercado de trabalho, haja vista o momento de desarticulação dos direitos trabalhistas e desemprego crônico que caracteriza a atual fase do mundo do trabalho no sistema capitalista ${ }^{10,11}$. Mesmo que para o profissional médico este comportamento do mercado de trabalho não apresente o mesmo panorama de crise, como no mercado de trabalho de outras categorias de profissionais de saúde, a exemplo de odontólogos ${ }^{10} \mathrm{e}$ enfermeiros ${ }^{11}$, a categoria médica também tem sofrido alterações nas características de seu mercado de trabalho, perdendo em parte a autorregulação de sua atividade, assim como autonomia $^{12}$, sendo que o trabalho assalariado no serviço público se torna importante também para estes profissionais.

Neste cenário, a inserção na Estratégia de Saúde da Família (ESF) emerge como oportunidade de emprego nas categorias profissionais inseridas oficialmente nesta estratégia proporcionalmente à crescente expansão dos postos de trabalho neste programa, desde o seu estabelecimento como principal modelo da atenção primária no Brasil. A Estratégia de Saúde da Família surge como proposta em 1994, sendo desenhada no sentido da reorientação e reorganização da atenção primária à saúde, dirigindo o processo de trabalho para a ação integrativa, integradora e pautada na complexidade das relações sociossanitárias e culturais do usuário do sistema, junto a seu entorno. Este enfoque, pautado no conceito de complexidade das relações entre homem-homens-mundo, se refletirá, de certa forma, na proposta integradora do processo de trabalho das diversas categorias em uma configuração idealizada pela equipe multiprofissional, a qual tem como objetivo o diagnóstico, o planejamento e o fazer integrado, fugindo da dimensão especializada e centrada na individualidade de cada categoria profissional no processo terapêutico. No entanto, incluindo o médico, os profissionais inseridos na ESF não fogem desta formação dicotômica com seus princípios ${ }^{2}$

No contexto da Amazônia Legal, em termos financeiros, o trabalho em Saúde Coletiva, em especial a ESF, constitui a opor- 
tunidade mais atrativa para os médicos, principalmente para recém-formados, em decorrência da remuneração paga pelo sistema público dos municípios distantes das capitais, mais alta quando comparada à de outras localidades do País. Uma vez que a dificuldade de acesso geográfico e, por consequência, a estrutura urbana e mercantil configuram uma realidade na região, existem problemas na fixação do médico nessas localidades, oferecendo aos profissionais recém-formados maior oportunidade de se inserir nesses postos de trabalho.

Segundo dados do Ministério da Saúde ${ }^{13}$, em 2007, os serviços de atenção primária responderam pela cobertura de $62 \%$ da população do Estado do Amazonas, sendo que a ESF apresenta uma cobertura total de $41 \%$, configurando-se como o principal modelo de atenção primária à saúde no Estado. Tendo em vista esse contexto, é muito importante estudar a formação dos médicos no Estado do Amazonas em Saúde Coletiva e para atuarem no SUS, dado implicar como a Equipe de Saúde da Família atuará em decorrência da importância que cada um dos profissionais ganha na dimensão do trabalho multiprofissional a que a ESF se propõe.

\section{ASPECTOS METODOLÓGICOS}

Esta investigação pautou-se na perspectiva qualitativa ${ }^{14-21}$, intencionando investigar o contexto da formação dos acadêmicos do curso de Medicina de uma universidade pública do Estado do Amazonas com relação a seu conhecimento sobre o trabalho médico em ESF, que será uma importante oportunidade para os primeiros vínculos trabalhistas destes futuros médicos, principalmente na Amazônia Legal. A escolha da instituição da qual se originam os entrevistados foi intencional, uma vez que numa instituição federal, por seu caráter público e diretamente vinculado ao Estado, depreende-se, em princípio, maior orientação para formar profissionais para a SUS, sobretudo numa região em que há escassez de profissionais, como a Amazônia Legal, em suas áreas não metropolitanas.

Foram utilizadas duas técnicas para coleta de informações: grupo focal ${ }^{16-18}$ e entrevistas individuais a partir de roteiro semiestruturado ${ }^{18-21}$, de forma a garantir tanto a percepção sobre a realidade com informações dinâmicas e de caráter coletivo, como informações referentes à perspectiva individual daqueles que vivenciaram o processo.

O trabalho no grupo focal enfocou a perspectiva construída na vivência do modelo pedagógico da graduação, particularmente nos aspectos referentes à prática em Saúde da Família. Individualmente, as entrevistas imergiram nos temas atuação do profissional na ESF, relação profissional-paciente e relação entre modelo pedagógico em Medicina e Saúde Coletiva. Todas as informações foram registradas por meio do uso de gravador com fita magnética.

Os critérios de inclusão dos sujeitos no presente estudo foram os seguintes: a) ser discente da universidade pública investigada; b) cursar o último período de Medicina; c) frequentar todo o curso na mesma turma que os outros sujeitos, já que era preciso que todos os depoentes tivessem sido submetidos ao mesmo currículo e processo de ensino-aprendizado. A inadequação a qualquer um destes critérios foi considerada como único critério de exclusão.

O grupo de sujeitos foi composto por oito acadêmicos de Medicina, número de acordo com o sugerido pela literatura pertinente ${ }^{19}$. Os mesmos acadêmicos foram convidados a contribuir na etapa das entrevistas individuais, que foram empreendidas conforme recomendado ${ }^{19-21}$.

O tratamento e a análise do produto empírico oriundo do grupo focal e das entrevistas individuais seguiram o processo preconizado pela literatura científica para análise hermenêutica dialética ${ }^{16-21}$, tendo por procedimentos: transcrição na íntegra de todos os registros; familiarização com o conteúdo por meio de leitura exaustiva; identificação da estrutura temática; indexação e recorte; sintetização e interpretação do produto final frente à literatura científica e documentos oficiais sobre o Sistema Único de Saúde e ESF ${ }^{22-38}$.

O presente estudo foi empreendido respeitando autorização do Comitê de Ética em Pesquisa da Universidade Federal do Amazonas (CEP/Ufam), conforme o protocolo 230/2006. Todo e qualquer procedimento de coleta de informação foi realizado após a leitura e explicação do Termo de Consentimento Livre e Esclarecido aos entrevistados, cujo consentimento em participar foi registrado com a assinatura por todos de duas vias do referido termo, ficando uma em sua posse e a outra em posse dos pesquisadores.

\section{SAÚDE COLETIVA, ESF E O MÉDICO QUE SE CONSTRÓI NA UNIVERSIDADE}

Na acepção dos entrevistados, o Sistema Único de Saúde (SUS) é uma área de atuação presente no cotidiano do médico, principalmente dos recém-formados, que buscam a remuneração segura pela grande oferta de postos de trabalho em diversas cidades do interior do Estado do Amazonas.

Para estes acadêmicos, tal atuação está impregnada de dificuldades, em termos tanto de formação específica, como de atividades peculiares a este campo, como a necessidade de conhecimento da estrutura legal e do funcionamento do SUS - segundo os depoentes, trata-se de um conhecimento deficitário para a maioria dos trabalhadores do setor público de saúde. Eles apon- 
tam que a saúde tem componentes mais amplos que a doença em si e que o campo epistemológico da Saúde Coletiva preconiza o enfoque nestes. Entretanto, opinam que a graduação não permite que consigam aplicar a Epidemiologia e seus métodos à prática cotidiana, silenciando sobre as ciências sociais e humanas em saúde, assim como o Planejamento e Gestão, campos que, junto com o primeiro, constituem parte da Saúde Coletiva.

Em particular, a ESF foi apresentada como um modelo que enfoca o vínculo entre o paciente e o médico, embora os entrevistados, de forma individual ou coletiva, nos grupos focais, tenham se apresentado em outra polaridade quanto a esta questão: esse vínculo foi tomado como ideal, mas um ideal não aplicável à prática clínica cotidiana, mesmo na própria ESF. E a principal razão desse posicionamento está ligada ao tempo e à aproximação necessários para estabelecer este vínculo.

Mesmo na ESF, as questões tempo de consulta e enfoque na doença foram centrais, de forma que o médico não consegue estabelecer o vínculo em razão da grande demanda por atendimento, o que ocasiona a dificuldade de se relacionar de maneira próxima ao paciente.

Outra dimensão da problemática apresentada é que o médico não é formado para a infraestrutura com que se deparará no serviço público, de modo que há certa inadequação tanto desta estrutura como da formação médica para a mesma, o que prejudicaria a formação do médico para a ESF.

Quanto à formação do médico para a ESF, tanto na percepção coletiva, quanto nas impressões individuais deste grupo, a estratégia demanda uma atuação médica que ultrapassa a atuação clínica sobre a doença, dado que permite a imersão nos determinantes de saúde da comunidade. Houve silêncio a respeito de como operacionalizar esta atuação, indício de que se trata de uma percepção tão-somente teórica, apesar de reconhecerem as implicações da complexidade da vida do paciente em sua saúde e a possibilidade de estes profissionais intervirem nessa complexidade, sobretudo na Estratégia de Saúde da Família.

O discurso de que a atuação na ESF extrapola os limites tradicionais e remodela o processo de trabalho dos profissionais que atuam neste modelo de atenção emergiu em diversos momentos, tanto nas discussões coletivas dos grupos focais, como nas entrevistas individuais de todos os sujeitos. Contraditoriamente a esta nova perspectiva no processo de trabalho, a figura médica é mencionada como detentora de status superior ao dos demais profissionais da equipe, havendo centralidade na atuação do médico à frente dos demais.

\section{O TRABALHO EM EQUIPE NA ESF}

Particularmente nos discursos coletivos, os entrevistados sustentaram que há necessidade de interdependência de todos os profissionais para uma perfeita atuação em Saúde da Família frente à complexidade das interações entre os determinantes de saúde, demandando um trabalho em equipe multiprofissional, multi e interdisciplinar. Tal discurso se mostrou vazio quando confrontado com o silêncio dos depoentes acerca de como operacionalizar estes conceitos, assim como diante da falta de debate sobre a articulação do SUS com a iniciativa privada por meio dos convênios, ou mesmo da ausência de menção quanto a como operacionalizar a necessidade de enfrentamento e articulação intersetorial da equipe de saúde de família, tanto em seu seio, como com setores que não fazem parte da saúde, mas estão implicados na vida dos pacientes.

A atuação do médico segundo os ideais da ESF foi encaminhada ao interferir no contexto complexo da vida dos pacientes. Este processo se inicia na relação interpessoal com o profissional, o qual, a partir desta abertura inicial, em teoria, deveria conhecer o entorno sociocultural e os determinantes de saúde para, num segundo momento, partir para o enfrentamento como profissional. E mais: como profissional de saúde.

Neste sentido, as necessidades de saúde dos pacientes atendidos na ESF foram mencionadas como oriundas de dinâmicas intersetoriais que, em teoria, os profissionais de ESF deveriam levar em conta durante sua atuação no modelo. No entanto, novamente, o grupo silenciou coletiva e individualmente sobre como operacionalizar esta atuação.

\section{SILÊNCIOS E CONTRADIÇÕES: INDÍCIOS DO DESPREPARO PARA ATUAÇÃO EM ESF}

Nos discursos acerca da prática em Saúde Coletiva, a fragilidade deste discurso "politicamente correto" em época de SUS foi mais evidente.

De maneira contundente, foi dito que o paciente que recorre ao serviço público de saúde não paga pelo atendimento e deve se contentar com o que lhe é oferecido; e que o médico não cursa Medicina para exercer a prática na atenção primária, seu lócus privilegiado é o hospital particular.

As demandas do SUS apontadas pelo campo da Saúde Coletiva e as dificuldades de funcionamento da ESF segundo o modelo preconizado pelo Ministério da Saúde tiveram soluções encaminhadas em direção à retirada de responsabilidade do médico nesse contexto. Assim, os problemas no trabalho em equipe, a falta de soluções práticas e a falta de intersetorialidade das equipes são dimensões em que o controle social, o governo, os outros 
profissionais e a própria comunidade foram responsabilizados. Segundo os depoentes, o médico nada tem a ver com esses contextos, apesar de, na ESF em particular, estar imerso nestas questões; apesar de ser parte integrante da equipe mínima deste modelo; apesar de todo o discurso de imersão na realidade e entendimento do "outro" com seus determinantes de saúde e seu entorno sociocultural.

No enfrentamento específico das demandas dos pacientes na ESF, houve priorização da ação curativa e clientelista, enfocando a doença e a ação clínica reducionista segundo um modelo biomédico, totalmente medicalizado. Embora o modelo preconize vínculo, e os próprios depoentes apontem a importância do mesmo, a dialogicidade entre médico e paciente não foi mencionada. Assim como também se silenciou sobre a horizontalidade e negociação entre as partes envolvidas no processo terapêutico, caracterizando uma postura tradicional, incoerente com o perfil do profissional para ESF, principalmente referindo-se às ações do médico no paciente, e não do médico junto com o paciente - postura que demanda um entendimento maior sobre a complexidade do processo saúde-doença no contexto do entorno sociocultural do paciente.

Nada foi mencionado com relação ao preparo para lidar com questões de saúde, características e limitações do sistema de saúde no interior do Estado do Amazonas. Isto pode constituir um indício de que, mesmo numa instituição pública e numa região que demanda articulações no âmbito dos serviços de saúde muito diferentes do que ocorre no restante do País, a formação destes futuros médicos se deu desarticulada dos aspectos de Saúde Coletiva da região e não priorizou o atendimento nos serviços de atenção primária.

\section{DISCUSSÃO}

Não apenas no contexto investigado, a atuação do médico em Saúde da Família apresenta dificuldades em se pautar conforme o preconizado para o modelo. O enfoque do trabalho ainda se baseia no conceito biomédico, inadequado à estratégia por diversos aspectos, incluindo a atenção fragmentada em especialidades ${ }^{39}$ e o atendimento desumanizado. Tais evidências emergem nas falas dos entrevistados no presente trabalho.

Falar de desumanização neste artigo é referir-se à prática da medicina como a violação do homem e de sua humanidade não apenas nos aspectos clínicos, mas também por não se considerarem as demandas dos pacientes que não as explicitamente biológicas, perspectiva que o ensino tradicional em medicina não permite ${ }^{40}$.

O médico tem dificuldade de se articular num trabalho interdisciplinar, necessário à perfeita consecução da ESF, não apenas por sua deficiência no processo formativo, mas também pelo silêncio quanto a como operacionalizar a interdisciplinaridade mesmo em documentos oficiais sobre o trabalho em Saúde da Família $^{41}$, o que aparece de maneira contundente nas falas dos entrevistados.

Estas questões levam à perspectiva de como está construído nestes acadêmicos o modelo de atuação profissional. Em estudo sobre a conceituação do "ser médico" por alunos de graduação e de residência médica, o significado dessa expressão foi apontado como o profissional que prima por aspectos técnicos e humanos na atividade profissional, mas com uma visão idealizada e longe da operacionalização ${ }^{40}$, de forma que o médico preconizado - tal qual a ESF recomenda - continua a ser um conceito muito próximo dos tipos ideais, mas, em termos práticos, não se mostra presente no dia-a-dia em Saúde da Família.

A legislação que regulamenta a prática em Saúde da Família também dificulta a prática segundo o que é idealizado para o programa. Isto porque as diretrizes de atuação médica de 1997 do Ministério da Saúde recomendam o generalista, que tem como competência atender todas as famílias, havendo certo retrocesso quando novas diretrizes, em 2000, sobrepõem este profissional à figura do clínico geral. Há problemas com a adequação da atuação médica na ESF, uma vez que esta se propõe implementar práticas substitutivas às práticas tradicionais em saúde. A atuação médica deveria, portanto, se pautar em novos modelos, não simplesmente no retorno à prática da clínica geral, que tem o conhecimento básico de todas as especialidades ${ }^{39}$.

O médico de Saúde da Família deveria se apresentar como um profissional com características de atuação diferentes das do médico tradicional das outras especialidades, da mesma forma no sentido técnico científico-restrito, mas de forma diferenciada no sentido amplo das relações sociais e da postura frente ao usuário ${ }^{39}$.

A formação médica é exígua em fundamentos e na construção de valores humanísticos junto aos acadêmicos, com parco entendimento de conhecimentos das ciências humanas. Isto acarretará problemas para o enfrentamento de um usuário que não se trata somente de um corpo biológico submetido à causalidade da doença, mas, sim, de um usuário inserido num entorno complexo e sociocultural do processo saúde-doença. Neste sentido, o campo epistemológico da Saúde Coletiva poderia contribuir muito com a formação médica, sendo preciso consolidar conhecimentos e habilidades da relação profissional-usuário que estejam de acordo com os pressupostos amplos, ou seja, um mínimo para o desenvolvimento do processo de humanização ${ }^{42}$.

Assim como o Estado promoveu diversas mudanças no sistema público de Saúde com a criação e a implementação do SUS, 
têm sido provocadas inúmeras ações para reorientar o talhe formativo em Medicina, direcionadas à formação para o SUS. O exemplo mais contundente é o Programa Nacional de Reorientação da Formação Profissional em Saúde (Pró-Saúde), que atinge não apenas a Medicina, mas também a Odontologia e a Enfermagem, visando transformar o processo de formação e geração do conhecimento para integrar o processo de ensino em saúde com a rede de serviços públicos. Neste processo, planejam-se impactos que levem à abordagem integral do processo saúde-doença pelos profissionais egressos, com a finalidade de responder as necessidades da população brasileira e operacionalizar o SUS conforme preconizado ${ }^{43}$.

OPró-Saúde está em fase de implementação. Paralelamente, as mudanças curriculares empreendidas por outras iniciativas nos currículos médicos não resultaram, até o presente, em mudanças de atitudes significativas, inclusive porque a mudança na estrutura curricular está longe de implicar uma mudança da postura docente ou mesmo nos estudantes de Medicina ${ }^{2,342}$. Neste sentido, há exiguidade de formas avaliativas sobre habilidades e atitudes dos acadêmicos de Medicina para oSUS, o que dificulta o processo de avaliação do ensino de graduação médica ${ }^{44}$.

Esta formação é dicotômica com relação a dois princípios necessários à consecução da ESF: o acolhimento ao usuário e o vínculo entre o profissional e este último, na medida em que implicam desde a oferta de ações clínicas e serviços, até a satisfação do usuário. Em sentido mais amplo, estes princípios essenciais ao processo de trabalho em ESF estimulam a autonomia e a cidadania, uma vez que a prestação de serviços de saúde passa a ser um ato que exige contraparte e participação do usuário. É essencial que o médico atue a partir da inclusão social e do compromisso com a vida dos cidadãos sob sua ação, e não apenas prescrevendo e ditando mudanças ${ }^{45}$.

Não apenas a formação do profissional médico está implicada na inadequação da atenção primária para com seus princípios norteadores. Sá ${ }^{46}$ discute que, no processo de implementação do SUS, diversas dimensões estão implicadas, incluindo questões políticas e sociais mais amplas. Tais dimensões limitam teórica e tecnicamente a consecução do processo como idealizado pelos princípios de reforma sanitária, culminando nas referidas contradições na prática cotidiana dos serviços de saúde no País, contexto que permeia a formação dos profissionais de saúde.

Há que se nortear a construção do futuro médico na contramão do aparente déficit nos aspectos humanos nos serviços de saúde, da exacerbação do individualismo e da exclusão social, características das sociedades contemporâneas que se reproduzem no íntimo da prestação de serviços de saúde no Brasil ${ }^{46}$. É preciso caminhar na direção contrária à deterioração da qualidade dos serviços, diminuindo a "banalização" da dor e do sofrimento dos usuários, pensamentos coerentes com a proposta da Saúde da Família. Nesta ação, os médicos e demais profissionais necessitam de capacitação e treino pautados nos princípios da atenção primária à saúde e nos novos espaços de atuação propiciados pela ESF, tal como a possibilidade de ação intersetorial junto à educação e aos demais setores/atores da vida da comunidade.

A centralidade da atuação do médico no processo de trabalho nas Unidades Básicas de saúde tradicionais se organiza de forma verticalizada e não permite atuação em equipe, uma vez que o trabalho dos demais profissionais se estabelece a partir da primeira categoria, divisão do trabalho própria da lógica da especialidade e do trabalho uniprofissional. Apesar da polarização quanto aos princípios da ESF, esta é a orientação da formação médica refletida nos achados do presente estudo. Ela está em consonância com outros trabalhos que indicam a organização fragmentada dos trabalhadores de saúde no processo terapêutico, em que, não havendo o trabalho processualmente oriundo da interação dos diversos profissionais, não haverá compromisso do médico com um resultado integrado e integrador junto aos demais profissionais ${ }^{47,48}$.

A literatura ressalta a necessidade de o profissional da atenção primária ter um conhecimento mais abrangente do que o profissional dos níveis secundário e terciário, tanto em termos de articulação dos problemas, como pela pouca utilização de recursos que auxiliam no diagnóstico, tais como exames ${ }^{1}$.

Outra dificuldade existente para os médicos é a utilização de ações preventivas e de cunho coletivo/comunitário, fugindo da lógica da especialização e da fragmentação, característica dos demais níveis de atenção. Nesse sentido, frente aos resultados do presente trabalho, há que se pensar em quanto a formação do médico na atualidade encaminha para os níveis secundário e terciário da atenção, quando deveria se pautar principalmente no nível primário. Esta lógica se torna ainda mais contundente na articulação com a realidade do Estado do Amazonas, em que os médicos que atuarão no interior exercerão sua profissão junto à atenção primária, geograficamente distante de qualquer unidade de atenção secundária ou terciária.

Pensando em termos de integralidade, ressalta-se a dificuldade do enfoque médico nessa realidade geográfica, em que a visão holística perpassará os determinantes sociais, epidemiológicos, sanitários e de saúde ambiental, entre outros, além da necessidade da efetiva referência e contrarreferência. No depoimento dos futuros médicos entrevistados, que estavam se formando numa instituição de ensino superior pública no Estado 
do Amazonas, existem grandes lacunas na perspectiva holística do processo terapêutico. Em especial, o usuário não foi percebido como sujeito desse processo, mas como alguém que deve seguir os ditames prescritos pelo médico.

Na medida em que a história da implantação do modelo de Saúde da Família no Brasil se pauta na tentativa de efetivar o SUS em seus princípios, a reorientação do modelo assistencial e a gestão do serviço planejada in loco deveriam estar implicadas na formação do médico ${ }^{48}$. Nesta lógica, a prática médica que não perpasse a abordagem integral e integrativa do cuidado em saúde dos usuários se aproxima da prática fragmentária, reducionista e biologicista - própria do modelo biomédico que caracteriza a ação tradicional do médico nas Unidades Básicas de Saúde. Uma atuação de características dicotômicas com a perspectiva totalizante e ampla, inerente à proposta da ESF e que, de longe, pede atuação médica muito além da resposta baseada em protocolos clínicos que respondam apenas ao sofrimento manifesto do usuário ${ }^{50}$.

Muito ainda há que se caminhar no sentido de uma formação médica que possibilite aos profissionais a integração com outras categorias de saúde, tanto nas ações preventivas, como nas de promoção e de assistência à saúde, enfocando a visão integral e integradora do usuário em seu entorno sociocultural, fugindo do modelo formativo e assistencial ainda vigente e hegemônico, que prima pela cura da doença, abordando o aspecto biológico do indivíduo, reduzindo-o à queixa clínica e limitando a atuação médica à prescrição medicamentosa, quando muito à orientação quanto à mudança de alguns poucos hábitos ${ }^{50}$.

Neste sentido, o campo epistemológico da Saúde Coletiva, com seus três pilares - Epidemiologia; Ciências Sociais/Humanas; Gestão/Planejamento -, possui ampla fundamentação, capaz de contribuir para uma nova formação médica, adequada aos anseios de políticas públicas como o Pró-Saúde e necessária à implementação doSUS de fato, em especial por possibilitar um alicerce teórico-conceitual para um novo talhe formativo, focado na atenção à saúde por um projeto contra-hegemônico ao talhe biomédico que ainda vigora. Mas para isso é necessário envolver o acadêmico desde a graduação no serviço com que se deparará no futuro e com uma formação cidadã, que integre o trabalho em que a equipe constitua um espaço interdisciplinar de fato. Não apenas no discurso, que se torna frágil quando se observam disparidades como as que emergiram no presente trabalho, como a de se formar numa universidade pública na Amazônia e pouco saber sobre SUS e muito menos ainda sobre a realidade da população da Amazônia.

\section{CONSIDERAÇÕES FINAIS}

A formação do profissional de saúde - em especial do médico - deve ser discutida amplamente com vistas a mudanças profundas ${ }^{19,12,26}$. A exclusão no acesso da população aos serviços e outras lacunas em Saúde Coletiva no Estado do Amazonas perderiam força por meio da existência de uma ESF em consonância com aquela preconizada - realidade não exclusiva do Amazonas, mas particularmente contundente nessa região.

Em termos contemporâneos, mais do que formar em instrumentos utilizados pela Saúde Coletiva, é necessário formar o médico para o trabalho imerso na vida do usuário e na atuação em equipe multiprofissional. Ele deve ser dotado da capacidade de estabelecer o diálogo, a troca e a construção de experiências integrativas, não só no âmbito dos profissionais de saúde, como na interface com os demais setores implicados na saúde dos usuários. Para isto, é preciso modificar a formação médica, pois como aprender a se articular se o aprendizado se dá fragmentado em especialidades?

\section{REFERÊNCIAS}

1. Campos CEA. O desafio da integralidade segundo as perspectivas da vigilância da saúde e da saúde da família. Cien Saude Colet. 2003;8(2):569-584.

2. Moretti-Pires RO. O pensamento crítico social de Paulo Freire sobre humanização e o contexto da formação do enfermeiro, do médico e do odontólogo. Ribeirão Preto; 2008. Doutorado [Tese] - Universidade de São Paulo.

3. Ronzani TM. A Reforma Curricular nos Cursos de Saúde: Qual o Papel das Crenças? Rev Bras Educ Med. 2007;31(1) 38-43.

4. Kemp A, Edler FC. A reforma médica no Brasil e nos Estados Unidos: uma comparação entre duas retóricas. Hist Cienc Saude Manguinhos. 2004;11(3): 569-85.

5. Franco TB, Bueno WS, Merhy EE. O acolhimento e os processos de trabalho em saúde: Betim, Minas Gerais, Brasil. Cad Saude Publica. 1999;2(15):345-53.

6. Merhy EE. Em busca do tempo perdido: a micropolítica do trabalho vivo em saúde. In: Merhy EE, Onocko R (org.). Agir em saúde: um desafio para o público. São Paulo: Hucitec; 1997. p. 71-112.

7. Merhy EE, Campos GWS, Cecílio LCO. Inventando a Mudança na Saúde. São Paulo: Hucitec; 1994

8. Alves Sobrinho EJ, Sousa MF. A tarefa: apagar os sinais vermelhos do PSF. In Sousa, MF (org.) Os sinais vermelhos do PSF. São Paulo: Hucitec; 2002. p.155-70. 
9. Markman Neto L. Políticas de Saúde, Sistema Único de Saúde e a prática do Programa de Saúde da Família num núcleo específico: limites e desafios. Ribeirão Preto; 2004. Mestrado [Dissertação] - Universidade de São Paulo.

10. Moretti-Pires RO. A mercantilização da saúde: o trabalho dos cirurgiões dentistas em um contexto de mudanças: estudo com cirurgiões dentistas assalariados do município de Ribeirão Preto (SP). Ribeirão Preto; 2005. Mestrado [Dissertação] - Universidade de São Paulo.

11. Pires D. Reestruturação produtiva e trabalho em saúde no Brasil. São Paulo, Annablume; 1998.

12. Machado MH. Os médicos e sua prática profissional: as metamorfoses de uma profissão. Rio de Janeiro; 1996. Doutorado [Tese] - Universidade Estadual do Rio de Janeiro.

13. Ministério da Saúde. DATASUS. Indicadores de Atenção Básica do Estado do Amazonas. [documento na internet]. Brasil; 200?. [acesso em 25 abr. 2008]. Disponível em: www.datasus.gov.br.

14. Ludke M, André MEAD. Pesquisa em Educação: abordagens qualitativas. 9ª ed. São Paulo: EPU; 2005.

15. Crotty M. The foundations of Social Research: meaning d perspective in the research process. London: Sage Publications; 2003.

16. Berg BL. Qualitative research methods for the social sciences. Long Beach - Califórnia, Ed.Pearson, 2004.

17. Beck CLC, Gonzales RMB, Leopardi MT. Técnicas e procedimentos de pesquisa qualitativa. In: Leopardi, M.T (org.). Metodologia da pesquisa na saúde. $2^{\underline{a}}$ ed. Florianópolis: Ed UFSC; 2002. p.223-44.

18. Morgan D. Focus Group as qualitative research. 2nd Ed. Sage Publications, 1997.

19. Minayo MCS. O desafio do conhecimento: pesquisa qualitativa em saúde. 6 6 ed. São Paulo: Hucitec; Abrasc; 1999.

20. Leopardi MT. Metodologia da pesquisa na saúde. $2^{\underline{a}}$ ed. Florianópolis: Ed. UFSC; 2002.

21. Haguette TMF. Metodologias qualitativas na sociologia. $8^{\mathrm{a}}$ ed. Petrópolis: Vozes, 2001.

22. Elias PE. Estado e Saúde: os desafios do Brasil contemporâneo. São Paulo Perspect. 2004;18(3):41-6.

23. Campos CEA. O desafio da integralidade segundo as perspectivas da vigilância da saúde e da saúde da família. Cienc Saúde Colet. 2003;8(2): 569-84.

24. Cohn A, Elias PE. Saúde no Brasil: políticas e organização de serviços. São Paulo: Cortez;Cedec;1998.

25. Silva PLB. Serviços de saúde: o dilema do SUS na nova década. São Paulo Perspect. 2003;17(1): 69-85.
26. Aciole GG. On pedagogical dimensions to citizenship building on social control practice. Interface Comun Saúde Educ. 2007;11(23):.409-26.

27. Campos CMS, Bataiero MO. Health needs: an analysis of Brazilian scientific literature from 1990 to 2004. Interface Comun Saúde Educ. 2007;11(23): 605-18.

28. Brasil. Ministério da Saúde. Secretaria de Gestão do Trabalho e de Educação na Saúde. Departamento de Educação na Saúde. Política de Educação e Desenvolvimento para o SUS. Caminhos para a Educação Permanente em Saúde. Brasília - DF; 2004.

29. Alves VS. A health education model for the Family Health Program: towards comprehensive health care and model reorientation. Interface Comun Saúde Educ. 2005;9(16): 39-52.

30. Brasil. Ministério da Saúde. Saúde da Família: uma estratégia para a reorientação do modelo assistencial. Brasília; 1997.

31. Brasil. Ministério da Saúde. A implantação da Unidade de Saúde da Família. Brasília: Secretaria de Políticas de Saúde; Departamento de Atenção Básica; 2000.

32. Ramos DD, Lima MADS. Acesso e acolhimento aos usuários em uma unidade de saúde de Porto Alegre, Rio Grande do Sul, Brasil. Cad Saúde Pública. 2003;19(1):27-34.

33. Pinheiro R. As práticas do cotidiano na relação oferta e demanda dos serviços de saúde: um campo de estudo e construção da integralidade. In: Pinheiro R, Mattos RA. (Org.) Os sentidos da integralidade na atenção e no cuidado à saúde. Rio de Janeiro: IMS-UERJ; Abrasco; 2001. p.65-112.

34. Pinheiro R. Práticas de saúde e integralidade. In: Brasil. Ministério da Saúde. Experiências Inovadoras no SUS: relatos de experiências. Brasília, 2002. p.15.

35. Brasil. Ministério da Saúde. Experiências Inovadoras no SUS: relatos de experiências. Brasília; 2002.

36. Teixeira RR. O acolhimento num serviço de saúde entendido como uma rede de conversações. Pinheiro R, Mattos RA (Orgs.) Construção da integralidade: cotidiano, saberes e práticas em saúde. IMS-UERJ; Abrasco; 2003. p.89-111.

37. Tesser CD. Social medicalization (II): biomedical limits and proposals for primary care clinics. Interface Comunic Saúde Educ. 2006;10(20): 347-62

38. Gomes MCPA, Pinheiro R. Reception and attachment: integral practices in health care administration in large urban centers. Interface Comun Saúde Educ. 2005;9(17): 287-301. 
39. Avarenga LMCA. A prática médica no programa de saúde da família e sua contribuição para mudança no modelo tecnicoassistencial em saúde. São Paulo; 2005. Mestrado [Dissertação] - Universidade de São Paulo.

40. Taquette SR, Schramm, SRFR, Soares LL, Carvalho SV. Situações eticamente conflituosas vivenciadas por estudantes de medicina. Rev Assoc Med Bras. 2005;51(1): 23-8.

41. Mota RA, Martins CGM, Veras RM. Papela dos profissionais de saúde na política de humanização hospitalar. Psicologia em Estudo. 2006; 11(2): 323-330.

42. Troncon LEA, Colares MFA, Figueiredo JFC, Cianflone ARL, Rodrigues MLV, Piccinato CV, et al. Atitudes de Graduandos de Medicina em relação à aspectos relevantes da prática médica. Rev Bras Educ Med. 2003;27(1): 20-8.

43. Brasil. Ministério da Saúde. Pró-Saúde: programa nacional de reorientação da formação profissional em saúde. Brasília: Ministério da Saúde, 2005.

44. Troncon LEA, Colares MFA, Figueiredo JFC, Cianflone ARL, Rodrigues MLV, Piccinato CV, et al. Avaliação de uma reestruturação curricular na faculdade de medicina de Ribeirão Preto. Rev Bras Educ Med. 2004;28(2):145-155.

45. Bueno WS, Merhy EE. Os equívocos da NOB 96: uma proposta em sintonia com os projetos neoliberalizantes? [documento na internet] In: Conferencia Nacional de Saúde On-Line: uma proposta em contrução. [acesso em: 20 maio 2008]. Disponível em: http:/ /www.datasus.gov.br/cns/temas/NOB96/NOB96crit.htm.
46. Sá MC. Subjetividade e projetos coletivos: mal-estar e governabilidade nas organizações de saúde. Ciênc Saúde Colet. 2001;6(1):151-164.

47. Campos GWS. Considerações sobre a arte e a ciência da mudança. In: Cecilio LCO, organizador. Inventando a mudança na saúde. 2ª ed. São Paulo: Hucitec; 1997. p. 29-87.

48. Merhy EE, Chakkour M, Stéfano E, Santos CM, Rodrigues RA, Oliveira PCP. Em busca de ferramentas analisadoras das tecnologias em saúde. In: Merhy EE, Onocko R, (Org.). Agir em saúde: um desafio para o público. São Paulo: Hucitec; 1997. p. 113-50.

49. Schimith MD, Lima MADS. Acolhimento e vínculo em uma equipe do Programa Saúde da Família. Cad Saúde Pública. 2004;20(6):1487-1494.

50. Alves VS. A health education model for the Family Health Program. Interface Comun Saúde Educ. 2005;9(16):39-52.

\section{CONFLITO DE INTERESSES}

Declarou não haver

\section{ENDEREÇO PARA CORRESPONDÊNCIA}

Universidade Federal do Amazonas - Instituto de Saúde e Biotecnologia

Estrada Coari-Mamiá, 305

Centro - Coari

CEP.: 69460-000 AM

E-mail: rodrigomoretti@ufam.edu.br 